\title{
Analysis of Single Nucleotide Polymorphisms in HLA-DRA, IL2RA, and HMGB1 Genes in Multiple Sclerosis
}

\author{
Mohsen Asouri ${ }^{1,2}$, Hamid Alinejad Rokni ${ }^{3}$, Mohammad Ali Sahraian ${ }^{4}$, \\ Sadegh Fattahi ${ }^{2}$, Nima Motamed ${ }^{5}$, Rozita Doosti ${ }^{4}$, Hamzeh Rahimi ${ }^{1}$, \\ Maryam Lotfi' ${ }^{2}$, Azam Moslemi ${ }^{6}$, Morteza Karimpoor*1, \\ Fereidoun Mahboudi*1, Haleh Akhavan-Niaki*7,8
}

\begin{abstract}
Background: Multiple sclerosis (MS) is a common demyelinating neurodegenerative disorder with significant heritability. Previous studies have associated genetic variants in human leukocyte antigen (HLA) complex, IL2RA, and HMGB1 genes with the pathophysiology of MS.

Methods: In order to investigate the gene association in the Iranian population, we performed a genotyping study of 36 variants in the mentioned genes using Sanger sequencing in 102 MS patients and 113 healthy controls.

Results: Our results identified significant associations as well as significant allele frequency differences in some of the studied single-nucleotide polymorphisms including rs4935356, rs3177928, and rs7197 from $H L A-D R A$ gene, and rs12722489 and rs12722490 variants from IL2RA gene ( $p<0.05)$. Moreover, the strong linkage disequilibrium of two common haplotypes was estimated from the HLA-DRA gene.

Conclusions: This association study may suggest the role of these polymorphisms in the genetic susceptibility of MS in the Iranian population and would facilitate the recognition of causative variants in this disease.
\end{abstract}

Keywords: $H M G B 1, H L A-D R A, I L 2 R A$, Multiple sclerosis, Polymorphism.

\section{Introduction}

Multiple sclerosis (MS) is the most common autoimmune neurologic disease which affects approximately two million new cases worldwide, especially young adults (1). Three patterns of disease are seen in MS patients: relapsing-remitting (RRMS), in which episodic exacerbations are separated by periods of recovery; secondary progressive (SPMS), people diagnosed with
RRMS eventually develop progressive disability; and primary progressive (PPMS), in which disability progresses steadily from disease onset (2). Multiple sclerosis arises when a susceptible individual encounters environmental triggers that stimulate an inflammatory response against selfantigen in the central nervous system (CNS). The immunological studies have shown that dis-

1: Department of Molecular Medicine, Biotechnology Research Center, Pasteur Institute of Iran, Tehran, Iran.

2: North Research Center, Pasteur Institute of Iran, Amol, Iran.

3: Systems Biology and Health Data Analytics Lab, the Graduate School of Biomedical Engineering, UNSW Sydney, 2052, NSW, AU.

4: Multiple Sclerosis Research Center; Neuroscience Institute, Tehran University of Medical Sciences, Tehran, Iran.

5: Department of Social Medicine, Zanjan University of Medical Sciences, Zanjan, Iran.

6: Department of Biostatistics, Faculty of Medical Sciences, Arak University of Medical Sciences, Arak, Iran.

7: Zoonoses Research Center, North Research Center, Pasteur Institute of Iran, Amol, Iran.

8: Department of Genetics, Faculty of Medicine, Babol University of Medical Sciences, Babol, Iran.

*Corresponding authors: Morteza Karimpoor; Tel: +98912 280 6133; E-mail: mortezakarimi@ pasteur.ac.ir

\& Fereidoun Mahboudi; Tel: +98912 280 6133; E-mail: mahboudi@pasteur.ac.ir

\& Haleh Akhavan-Niaki; Tel: +98911 125 5920; E-mail: halehakhavan@yahoo.com.

Received: 3 Feb, 2020; Accepted: 23 Feb, 2020 
regulation of cellular and humoral immune responses elicit infiltration of lymphocytes and macrophages into the CNS along with anti-myelin antibodies and complement activation, which leads to episodes of inflammatory demyelination and a progressive neurodegenerative process with axonal transection and neuronal loss as an early event (3). An eruption of focal inflammation is responsible for the episodic RRMS phase, while axonal loss and neurodegeneration cause progressive accumulation of disability $(4,5)$.

Both environmental and genetic factors are involved in the etiology of MS. Vitamin D deficiency, smoking, Ultraviolet B (UVB)/ sunshine, and some pathogens are also considered as environmental risk factors for the development of MS (6).

Genetic factors are primarily responsible for the increased frequency of the disease in the relatives of affected individuals. Studies on twins and siblings suggest that multiple genes, each exerting different effects, play considerable roles in susceptibility to MS. Therefore, MS is considered as a mutagenic and complex disorder. Candidategene studies have validated the human leukocyte antigen (HLA) class II (HLA-DRBI) as the strongest susceptibility locus for MS. In 2011, the genome-wide association studies (GWAS) and ImmunoChip studies with more than 9,000 MS cases discovered 110 non-HLA genetic loci associated with MS (7).

Further studies have shown that genes encoding high mobility group box protein 1 (HMGBI) and the IL-2 receptor $\alpha$ chain $(I L-2 R \alpha)$ exert critical functions regarding immune responses in the development of neurodegenerative diseases, such as Alzheimer's disease, Parkinson's disease, and MS (8). Elevation of peripheral blood mononuclear cells (PBMC), as well as upregulated serum and cerebrospinal fluid (CSF) levels of $H M G B 1$, have been implicated in MS patients in several studies $(9,10)$. Large numbers of macrophages and microglia expressing the endogenous $H M G B 1$ and its ligands as well as Toll-like receptor 2 (TLR2) and TLR4 are also found in MS patients (11). The cytokine interleukin-2 (IL-2 has a wide range of effects that are essential for the balance between immune response and tolerance. The IL-2 receptor $\alpha$ chain (IL-2R $\alpha$ ), also known as CD25, is a central constituent of the trimeric IL-2 receptor complex that binds to IL-2. It has been shown that singlenucleotide polymorphisms (SNPs) in or near HLA$D R A, I L 2 R A$, and HMGB1 genes are associated with the increased risk of immune-mediated diseases including MS. The association of some SNPs in or near the mentioned genes has been studied extensively regarding increased risk of developing this disease. For instance, MSassociated IL2RA SNPs rs2104286 and rs11256593 are associated with CD25 expression on $\mathrm{CD} 4^{+} \mathrm{T}$ cells. Changes in CD25 expression may influence the immune and inflammation signaling cascades, thus affecting $\mathrm{CD} 4^{+} \mathrm{T}$ cell differentiation and $\mathrm{T}_{\text {Reg }}$ cells suppressive activity (12).

The purpose of this study was to investigate the frequency of 36 SNPs in three known loci, HLA$D R A$, IL2RA, and HMGBI in the Iranian population. A total of $102 \mathrm{MS}$ patients and 112 control subjects were selected and genotyped using polymerase chain reaction (PCR) method and Sanger sequencing.

\section{Materials and methods Sample collection}

A total of 102 MS patients and 112matched controls were selected from the individuals referred to Sina teaching Hospital in Tehran, Iran. The patients were diagnosed based on the McDonald criteria (4), clinical signs and symptoms; all results were confirmed using brain magnetic resonance imaging (MRI). The control group was selected from patients who attended the hospital due to other causes and brain MRI was used to rule out MS. This group was age and sexmatched with the case group. The Ethics Committees of Sina teaching Hospital and Pasteur Institute of Iran approved the study. Written informed consent was taken from all participants.

\section{DNA extraction}

Five $\mathrm{ml}$ of peripheral blood from patients and healthy controls were collected in K3-EDTA tube and genomic DNA was extracted and purified from whole-blood lymphocytes by Mini QIAamp DNA Mini Kits (Cat. 51104; Qiagen GmbH, Hilden, Germany) according to the manufacturer's instructions. The DNA measurement and quality control were performed using spectrophotometry 
observance at 260/280 and 260/230 nm, respectively. The DNA integrity and fragmentation were investigated using $1 \%$ agarose gel electrophoresis.

\section{Polymerase chain reaction (PCR)}

PCR was performed by using Step One Plus Realtime PCR system (Applied Biosystems, Foster City, USA) to detect the 36 polymorphisms. These SNPs, located in HLA-DRA, IL2RA, and HMGBI genes, were selected by Haploview 4.2 using genotype data from Genome Browser release \#27 in the HapMap Project (http://www.hapmap.org). The chromosome location, dbSNP number, and gene annotation of selected polymorphisms are summarized in Table 1. The specific primer sequences for each gene were designed through the Primer3 online software (http://frodo.wi.mit.edu/primer3), and the primer specificity was checked out by Primer-BLAST and SNPCheck V3 tools. The physiochemical properties of primers were further evaluated using Gene Runner software. All primer sequences are shown in Table 2.

Table 1. The genomic properties of studied polymorphism of $H M G B 1, H L A-D R A$, and IL2RA genes that were extracted from UCSC genome browser for investigated genomic regions in this study.

\begin{tabular}{|c|c|c|c|c|c|}
\hline Variant ID & Chr:bp & Allele & MAF & Location & Gene \\
\hline rs538493533 & $13: 30460237$ & $\mathrm{~A} / \mathrm{G}$ & 0.001 & $3^{\prime} \mathrm{UTR}$ & $H M G B 1$ \\
\hline rs577524260 & $13: 30460223$ & $\mathrm{C} / \mathrm{T}$ & 0.001 & 3'UTR & $H M G B 1$ \\
\hline rs111892138 & $13: 30460267$ & $\mathrm{~T} / \mathrm{C}$ & 0.022 & $3^{\prime} \mathrm{UTR}$ & $H M G B 1$ \\
\hline rs201945336 & $13: 30460287-8$ & AA/A & - & $3^{\prime} \mathrm{UTR}$ & $H M G B 1$ \\
\hline rs182881863 & 13:30460196 & $\mathrm{T} / \mathrm{G}$ & 0.001 & $3^{\prime} \mathrm{UTR}$ & $H M G B 1$ \\
\hline rs149637108 & 13:30460189-94 & СТТССТ/СТ & - & $3^{\prime} \mathrm{UTR}$ & $H M G B 1$ \\
\hline rs61338778 & $13: 30460263-5$ & TTT/ТTTT & - & $3^{\prime} \mathrm{UTR}$ & $H M G B 1$ \\
\hline rs55642413 & $13: 30460306-7$ & GG/G & - & $3^{\prime} \mathrm{UTR}$ & $H M G B 1$ \\
\hline Rs9281809 & $32444502 \& 32444503$ & -/АACTAACT & 0.344 & intron & $H L A-D R A$ \\
\hline Rs4935356 & $6: 32444611$ & $\mathrm{~T} / \mathrm{A} / \mathrm{G}$ & $0.341(\mathrm{~T})$ & Intron & $H L A-D R A$ \\
\hline Rs3135390 & $6: 32444618$ & $\mathrm{C} / \mathrm{A}$ & $0.131(\mathrm{C})$ & Intron & $H L A-D R A$ \\
\hline Rs4935354 & $6: 32444621$ & $\mathrm{C} / \mathrm{G} / \mathrm{T}$ & $0.341(\mathrm{C})$ & Intron & $H L A-D R A$ \\
\hline Rs3177928 & $6: 32444658$ & G/A & $0.120(\mathrm{~A})$ & 3'UTR & $H L A-D R A$ \\
\hline Rs7194 & $6: 32444703$ & G/A & $0.341(\mathrm{G})$ & 3'UTR & $H L A-D R A$ \\
\hline rs7195 & $6: 32444762$ & $\mathrm{~A} / \mathrm{G}$ & $0.341(\mathrm{~A})$ & 3'UTR & $H L A-D R A$ \\
\hline Rs1131541 & $6: 32444789$ & $\mathrm{~T} / \mathrm{A}$ & $0.120(\mathrm{~A})$ & 3'UTR & $H L A-D R A$ \\
\hline Rs7196 & $6: 32444794$ & $\mathrm{~A} / \mathrm{T}$ & $0.221(\mathrm{~A})$ & 3'UTR & $H L A-D R A$ \\
\hline Rs7197 & $6: 32444803$ & $\mathrm{~T} / \mathrm{C} / \mathrm{G}$ & $0.117(\mathrm{~T})$ & 3'UTR & $H L A-D R A$ \\
\hline Rs1051336 & $6: 32444815$ & $\mathrm{G} / \mathrm{A} / \mathrm{C}$ & $0.120(\mathrm{~A})$ & 3'UTR & $H L A-D R A$ \\
\hline Rs111471704 & $6: 32444889$ & $\mathrm{~T} / \mathrm{C}$ & - & 3'UTR & $H L A-D R A$ \\
\hline Rs1157343109 & $6: 32444988$ & $\mathrm{~T} / \mathrm{C}$ & - & 3'UTR & $H L A-D R A$ \\
\hline Rs1041885 & $6: 32445032$ & T/A & $0.119(\mathrm{~A})$ & 3'UTR & $H L A-D R A$ \\
\hline Rs12722489 & 10:6060049 & $\mathrm{C} / \mathrm{T}$ & $0.091(\mathrm{~T})$ & Intron & $I L 2 R A$ \\
\hline Rs917751277 & 10:6060048 & $\mathrm{A} / \mathrm{G}$ & - & Intron & IL2RA \\
\hline Rs992067421 & 10:6060043 & $\mathrm{C} / \mathrm{A}$ & - & Intron & $I L 2 R A$ \\
\hline Rs959264277 & 10:6060039 & $\mathrm{A} / \mathrm{G}$ & - & Intron & $I L 2 R A$ \\
\hline Rs11597542 & 10:6059981 & $\mathrm{T} / \mathrm{C}$ & $0.001(\mathrm{C})$ & Intron & $I L 2 R A$ \\
\hline Rs140860467 & 10:6059935 & $\mathrm{T} / \mathrm{C}$ & $0.007(\mathrm{C})$ & Intron & $I L 2 R A$ \\
\hline Rs17149458 & 10:6059897 & T/A & $0.029(\mathrm{~A})$ & Intron & $I L 2 R A$ \\
\hline Rs12722490 & 10:6059828 & $\mathrm{C} / \mathrm{T}$ & $0.010(\mathrm{~T})$ & Intron & $I L 2 R A$ \\
\hline Rs3118470 & 10:6059750 & $\mathrm{T} / \mathrm{A} / \mathrm{C}$ & $0.319(\mathrm{C})$ & Intron & $I L 2 R A$ \\
\hline Rs78556477 & 10:6059635 & G/A & $0.059(\mathrm{~A})$ & Intron & IL2RA \\
\hline Rs41294925 & 10:6059590 & $\mathrm{A} / \mathrm{G}$ & $0.008(\mathrm{G})$ & Intron & $I L 2 R A$ \\
\hline Rs12722491 & 10:6059467 & $\mathrm{G} / \mathrm{T}$ & $0.010(\mathrm{~T})$ & Intron & $I L 2 R A$ \\
\hline Rs550805995 & 10:6059430 & $\mathrm{G} / \mathrm{A}$ & $0.001(\mathrm{~A})$ & Intron & $I L 2 R A$ \\
\hline Rs12722621 & 10:6059210 & $\mathrm{C} / \mathrm{A}$ & $0.041(\mathrm{~A})$ & Intron & IL2RA \\
\hline
\end{tabular}


Table 2. Sequence and amplicon size of primers

\begin{tabular}{llll}
\hline \multicolumn{1}{c}{ Gene } & \multicolumn{1}{c}{ Forward } & \multicolumn{1}{c}{ Reverse } & Length (bp) \\
\hline IL2RA & ATGCTCTGCCTCTGGAAGACAC & TATCTCAATGGGTTTCCACACTGT & 1472 \\
$H L A-D R A$ & TGCCTGCTTTTGCTTCTTTAGTCTC & AGGTGGTTTCAAGAATCAGTCAGAC & 1173 \\
$H M G B I$ & AGAATGTATCCCCAAAAGCGTGAG & CACAGCACTGTAACTATCTTGGC & 1366 \\
\hline
\end{tabular}

DNA amplification was carried out by PCR with $3 \mu \mathrm{l}$ of DNA in a $25 \mu \mathrm{l}$ total reaction mixture containing $12.5 \mu \mathrm{l}$ master mix $(2 \mathrm{x})$ and $0.5 \mu \mathrm{M}$ of each primer. Thermal cycling conditions were as follows: denaturation at $94{ }^{\circ} \mathrm{C}$ for $5 \mathrm{~min}$, followed by 35 cycles of denaturation at $94{ }^{\circ} \mathrm{C}$ for $30 \mathrm{~s}$, annealing at $62{ }^{\circ} \mathrm{C}$ for $30 \mathrm{~s}$, and extension at 72 ${ }^{\circ} \mathrm{C}$ for $30 \mathrm{~s}$. The amplification was followed by the last extension step at $72{ }^{\circ} \mathrm{C}$ for $5 \mathrm{~min}$. The PCR products were monitored after electrophoresis with agarose gel $1.5 \%$ using a gel documentation system.

\section{Sanger sequencing}

The amplicons after gel purification were sent to the Macrogen Company (Seoul, South Korea) for Sanger sequencing. The results were trimmed and analyzed by BioEdit and Chromas software to ensure the quality and accuracy of the sequencing. Then, the extracted sequences were blasted against the non-redundant (NR) database to validate the annotated regions with sequences of interested genes.

\section{Statistical analysis}

The calculated findings were illustrated via reporting odds ratio (OR) and $95 \%$ confidence intervals (CI) for each SNP. The differences in allelic and genotypic distribution between the two studied groups were considered as significant if the computed $p$ value was less than 0.05. The Hardy-Weinberg equilibrium (HWE) was evaluated through the Chi-square $(\chi 2)$ goodness-of-fit test, and allele frequencies and genotype distributions were analyzed using the Chi-square $(\chi 2)$ test. Pairwise, linkage disequilibrium (LD) of HLA-DRA, IL2RA, and $H M G B 1$ SNPs was operated using Haploview 4.1 software and the obtained data were reported by describing the $\mathrm{D}^{\prime}$ and $\mathrm{r}^{2}$ parameters. Moreover, 10.000 permutations were conducted to address significant levels in haplotypes.

\section{Results}

In this study, $102 \mathrm{MS}$ patients, (17.6\% men and $82.4 \%$ women) with a mean age of $35.29 \pm 14.66$ years and 113 healthy controls $(24.8 \%$ men and $75.2 \%$ women) were compared regarding totally 36 polymorphisms within the HLA-DRA, IL2R, and $H M G B 1$ genes, 14, 14 and, 8, respectively, by PCR-sequencing method. The association between various polymorphisms of these three genes and MS disease was evaluated by the chisquare test. Thus, the frequency of related genotypes in each polymorphism was computed in MS patients and control groups, separately. No deviation from HWE was identified in both MS patients and control groups for all SNPs $(p>$ 0.05) (data not shown).

Based on our results, in the polymorphisms of the HLA-DRA gene, the rs4935356 ( $p=0.001)$, rs3177928 ( $p=0.002)$, and rs7197 $(p=0.002)$ SNPs were significantly associated with the risk of MS disease. Moreover, the frequencies of the A allele of rs3135390 $(p=0.026)$, an allele in rs3177928 $(p=0.027)$, and C allele in rs7197 ( $p=$ 0.001 ) were significantly altered in our MS patients. The findings of association analysis between HLA-DRA SNPs and the risk of MS are exhibited in Table 3.

Results of variants of IL2RA revealed that there was a statistically significant association between rs12722489 $(p=0.007)$ and rs12722490 ( $p=0.03$ ) SNPs and the risk of MS; however, other polymorphisms of IL2RA gene (rs917751277, rs992067421, rs959264277, rs11597542, rs140860467, rs17149458, rs3118470, rs78556477, rs41294925, rs12722491, rs550805995, and rs12722621) had no significant differences between the two studied groups $(p>0.05)$. The frequency of $\mathrm{T}$ allele in rs12722489 showed significant discrepancies in MS patients compared to healthy controls. Differences in other SNPs of this gene did not 
reach a level of significance (Table 4). Upon analysis of the polymorphisms of $H M G B 1$ gene, the genotype and allele frequencies of rs146076135, rs201945336, rs111892138, rs200308321, rs149637108, rs538493533, rs577524260, and rs182881863 SNPs were calculated and, finally no significant difference was observed in allele and genotype frequencies in both groups $(p>0.05)$ (Table 5). Finally, the linkage disequilibrium (LD) patterns of HLA-DRA, IL2RA, and HMGB1 SNPs were analyzed. Figure 1 illustrates the strong patterns of LD found in patients of this study. As shown, two haplotype blocks as following were significant predictors of MS disease in the HLA-DRA gene: one block of rs7194 and rs7195 SNPs; and another block consisting of rs4935356, rs3135390, rs4935354, rs3177928, rs7194, rs7195, rs1131541, rs7196, and rs7197 SNPs.

Table 3. The frequency of HLA-DRA alleles and genotypes in Iranian multiple sclerosis (MS) patients and controls.

\begin{tabular}{|c|c|c|c|c|c|}
\hline Polymorphism & Stat & & $\begin{array}{c}\text { (Frequency of Patients) } \\
\text { Percent }\end{array}$ & $\begin{array}{c}\text { (Frequency of Control) } \\
\text { Percent }\end{array}$ & P-value \\
\hline \multirow{9}{*}{ rs4935356 } & \multirow{3}{*}{ allele } & G & (143) 70.8 & (136) 65.4 & \multirow{3}{*}{0.219} \\
\hline & & $\mathrm{T}$ & (49) 24.25 & (53) 25.5 & \\
\hline & & $\mathrm{A}$ & (10 4.95 & (19) 9.1 & \\
\hline & \multirow{6}{*}{ genotype } & $\mathrm{A} / \mathrm{A}$ & (1) 1.0 & (0) 0.0 & \multirow{6}{*}{$<0.001$} \\
\hline & & G/A & (6) 5.9 & (15) 14.4 & \\
\hline & & $\mathrm{G} / \mathrm{G}$ & (52) 51.5 & (50) 48.1 & \\
\hline & & $\mathrm{G} / \mathrm{T}$ & (33) 32.7 & (21) 20.2 & \\
\hline & & T/A & (2) 2.0 & (4) 3.8 & \\
\hline & & $T / T$ & (7) 6.9 & (14) 13.5 & \\
\hline \multirow{7}{*}{ rs3135390 } & \multirow{3}{*}{ allele } & A & (170)84.15 & (191) 91.8 & \multirow{3}{*}{0.026} \\
\hline & & $\mathrm{C}$ & (31)15.35 & (17) 8.2 & \\
\hline & & $\mathrm{T}$ & (1) 0.5 & (0) 0.0 & \\
\hline & \multirow{4}{*}{ genotype } & $\mathrm{A} / \mathrm{A}$ & (74)73.3 & (89) 85.6 & \multirow{4}{*}{0.049} \\
\hline & & $\mathrm{C} / \mathrm{A}$ & (21) 20.8 & (13) 12.5 & \\
\hline & & $\mathrm{C} / \mathrm{C}$ & (5) 5.0 & (2) 1.9 & \\
\hline & & T/A & (1) 1.0 & (0) 0.0 & \\
\hline \multirow{5}{*}{ rs4935354 } & \multirow{2}{*}{ allele } & $\mathrm{C}$ & (49) 24.26 & (55) 26.4 & \multirow{2}{*}{0.611} \\
\hline & & $\mathrm{T}$ & (153) 75.74 & (153) 73.6 & \\
\hline & \multirow{3}{*}{ genotype } & $\mathrm{C} / \mathrm{C}$ & (7) 6.9 & (15) 14.4 & \multirow{3}{*}{0.17} \\
\hline & & $\mathrm{C} / \mathrm{T}$ & (35) 34.7 & (25) 24.1 & \\
\hline & & $\mathrm{T} / \mathrm{T}$ & (59) 58.4 & (64) 61.5 & \\
\hline \multirow{5}{*}{ rs3177928 } & \multirow{2}{*}{ allele } & $\mathrm{G}$ & (191) 94.55 & (184) 88.5 & \multirow{2}{*}{0.027} \\
\hline & & A & (11) 5.45 & (24) 11.5 & \\
\hline & \multirow{3}{*}{ genotype } & $\mathrm{A} / \mathrm{A}$ & (1) 1.0 & (0) 0.0 & \multirow{3}{*}{0.002} \\
\hline & & G/A & (9) 8.9 & (24) 23.1 & \\
\hline & & $\mathrm{G} / \mathrm{G}$ & (91) 90.1 & (80) 76.9 & \\
\hline \multirow{5}{*}{ rs7194 } & \multirow{2}{*}{ allele } & G & (49) 24.26 & (51) 24.5 & \multirow{2}{*}{0.951} \\
\hline & & A & (153) 75.74 & (157) 75.5 & \\
\hline & \multirow{3}{*}{ genotype } & $\mathrm{A} / \mathrm{A}$ & (59) 58.4 & (66) 63.5 & \multirow{3}{*}{0.24} \\
\hline & & G/A & (35) 34.7 & (25) 24.0 & \\
\hline & & $\mathrm{G} / \mathrm{G}$ & (7) 6.9 & (13) 12.5 & \\
\hline & & $\mathrm{G}$ & (153) 75.74 & (159) 76.4 & 0869 \\
\hline & allele & A & (49) 24.26 & (49) 23.6 & 0.868 \\
\hline rs7195 & & $\mathrm{A} / \mathrm{A}$ & (7) 6.9 & (10) 9.6 & \\
\hline & genotype & G/A & (35) 34.7 & (29) 27.8 & 0.63 \\
\hline & & $\mathrm{G} / \mathrm{G}$ & (59) 58.4 & (65) 62.0 & \\
\hline & & $\mathrm{T}$ & (189) 93.56 & (189) 90.9 & \\
\hline & allele & $\mathrm{A}$ & (13) 6.44 & (19) 9.1 & 0.308 \\
\hline rs1131541 & & $\mathrm{A} / \mathrm{A}$ & (0) 0.0 & (1) 1.0 & \\
\hline & genotype & T/A & (13) 12.0 & (17) 16.4 & 0.49 \\
\hline & & $\mathrm{T} / \mathrm{T}$ & (88) 87.1 & (86) 82.7 & \\
\hline s7196 & allele & $\mathrm{T}$ & (165) 81.7 & (171) 82.2 & 0.889 \\
\hline
\end{tabular}




\begin{tabular}{|c|c|c|c|c|c|}
\hline & & $\mathrm{A}$ & (37) 18.3 & (37) 17.8 & \\
\hline & \multirow{3}{*}{ genotype } & $\mathrm{A} / \mathrm{A}$ & (5) 5.0 & (6) 5.8 & \multirow{3}{*}{0.87} \\
\hline & & $\mathrm{T} / \mathrm{A}$ & (27) 26.7 & (25) 24.0 & \\
\hline & & $\mathrm{T} / \mathrm{T}$ & (69) 68.3 & (73) 70.2 & \\
\hline \multirow{5}{*}{ rs7197 } & \multirow{2}{*}{ allele } & $\mathrm{C}$ & (172) 85.1 & (198) 95.2 & \multirow{2}{*}{0.001} \\
\hline & & $\mathrm{T}$ & (30) 14.9 & (10) 4.8 & \\
\hline & \multirow{3}{*}{ genotype } & $\mathrm{C} / \mathrm{C}$ & (75) 74.3 & (95) 91.3 & \multirow{3}{*}{0.001} \\
\hline & & $\mathrm{C} / \mathrm{T}$ & (22) 21.3 & (8) 7.6 & \\
\hline & & $\mathrm{T} / \mathrm{T}$ & (4) 4.0 & (1) 1.0 & \\
\hline \multirow{4}{*}{ rs1051336 } & \multirow{2}{*}{ allele } & $G$ & (189) 93.56 & (191) 91.8 & \multirow{2}{*}{0.490} \\
\hline & & $\mathrm{A}$ & (13) 6.44 & (17) 8.2 & \\
\hline & \multirow{2}{*}{ genotype } & G/A & (13) 12.9 & (17) 16.3 & \multirow{2}{*}{0.57} \\
\hline & & $\mathrm{G} / \mathrm{G}$ & (88) 87.1 & (87) 83.7 & \\
\hline \multirow{4}{*}{ rs111471704 } & \multirow{2}{*}{ allele } & $\mathrm{T}$ & (201) 99.5 & (208) 100 & \multirow{2}{*}{0.493} \\
\hline & & $\mathrm{A}$ & (1) 0.5 & (0) 0.0 & \\
\hline & \multirow{2}{*}{ genotype } & $\mathrm{T} / \mathrm{A}$ & (1) 1.0 & (1) 1.0 & \multirow{2}{*}{0.99} \\
\hline & & $\mathrm{T} / \mathrm{T}$ & (99) 99.0 & (103) 99.0 & \\
\hline \multirow{2}{*}{ rs1157343109 } & allele & $\mathrm{T}$ & (202) 100 & (208) 100 & \multirow{2}{*}{-} \\
\hline & genotype & $\mathrm{T} / \mathrm{T}$ & (101) 100 & (104) 100 & \\
\hline \multirow{2}{*}{ rs1041885 } & allele & $\mathrm{T}$ & (202) 100 & (208) 100 & \multirow{2}{*}{-} \\
\hline & genotype & $\mathrm{T} / \mathrm{T}$ & (101) 100 & (104) 100 & \\
\hline
\end{tabular}

Table 4. The frequency of IL2RA allele and genotype in Iranian multiple sclerosis (MS) patients and controls.

\begin{tabular}{|c|c|c|c|c|c|}
\hline Polymorphism & \multicolumn{2}{|c|}{ Status } & $\begin{array}{c}\text { (Frequency of Patients) } \\
\text { Percent }\end{array}$ & $\begin{array}{c}\text { (Frequency of Control) } \\
\text { Percent }\end{array}$ & P-value \\
\hline \multirow{5}{*}{ rs12722489 } & \multirow[b]{2}{*}{ allele } & $\mathrm{A}$ & (28) 13.7 & (13) 5.8 & \multirow[b]{2}{*}{0.005} \\
\hline & & $\mathrm{G}$ & (176) 86.3 & (211) 94.2 & \\
\hline & \multirow{3}{*}{ genotype } & $\mathrm{AA}$ & (5) 4.9 & (3) 2.8 & \multirow{3}{*}{0.007} \\
\hline & & G/A & (18) 17.6 & (5) 4.7 & \\
\hline & & $\mathrm{G} / \mathrm{G}$ & (79) 77.5 & (99) 92.5 & \\
\hline \multirow{2}{*}{ rs917751277 } & allele & $\mathrm{T}$ & (204) 100 & (224) 100 & - \\
\hline & genotype & TT & $(102) 100$ & $(107) 100$ & - \\
\hline \multirow{2}{*}{ rs992067421 } & allele & $\mathrm{G}$ & (204) 100 & (224) 100 & - \\
\hline & genotype & GG & $(102) 100$ & $(107) 100$ & - \\
\hline \multirow{2}{*}{ rs959264277 } & allele & $\mathrm{T}$ & (204) 100 & (224) 100 & - \\
\hline & genotype & TT & $(102) 100$ & (107) 100 & - \\
\hline \multirow{2}{*}{ rs11597542 } & allele & $\mathrm{A}$ & (204) 100 & (224) 100 & - \\
\hline & genotype & AA & $(102) 100$ & (107) 100 & - \\
\hline \multirow{2}{*}{ rs 140860467} & allele & A & (204) 100 & (224) 100 & - \\
\hline & genotype & $\mathrm{AA}$ & $(102) 100$ & (107) 100 & - \\
\hline \multirow{2}{*}{ rs17149458 } & allele & $\mathrm{A}$ & (204) 100 & (224) 100 & - \\
\hline & genotype & $\mathrm{AA}$ & $(102) 100$ & (107) 100 & - \\
\hline \multirow{5}{*}{ rs12722490 } & \multirow{2}{*}{ allele } & $\mathrm{A}$ & (12) 5.9 & (7) 3.1 & \multirow{2}{*}{0.167} \\
\hline & & $\mathrm{G}$ & (192) 94.1 & (217) 96.9 & \\
\hline & \multirow{3}{*}{ genotype } & $\mathrm{AA}$ & (0) 0.0 & (1) 0.9 & \multirow{3}{*}{0.03} \\
\hline & & GA & (12) 11.8 & (5) 4.7 & \\
\hline & & GG & (90) 88.2 & (101)94.4 & \\
\hline \multirow{5}{*}{ rs3118470 } & \multirow{2}{*}{ allele } & $\mathrm{A}$ & (130) 63.7 & (150) 67.0 & \multirow{2}{*}{0.482} \\
\hline & & $\mathrm{G}$ & (74) 36.3 & (74) 33.0 & \\
\hline & \multirow{3}{*}{ genotype } & $\mathrm{AA}$ & (45) 44.1 & (52) 48.6 & \multirow{3}{*}{0.83} \\
\hline & & GA & (40)39.2 & (39)36.4 & \\
\hline & & GG & (17)16.7 & (16) 15.0 & \\
\hline \multirow{2}{*}{ rs78556477 } & allele & $\mathrm{C}$ & (204) 100 & (224) 100 & - \\
\hline & genotype & $\mathrm{CC}$ & 100 & 100 & - \\
\hline \multirow{3}{*}{ rs41294925 } & dlold & $\mathrm{T}$ & 98.5 & (224) 100 & \multirow{2}{*}{0.107} \\
\hline & allele & $\mathrm{C}$ & (3) 1.5 & (0) 0.0 & \\
\hline & genotype & $\mathrm{TC}$ & (3) 2.9 & (0) 0.0 & 0.114 \\
\hline
\end{tabular}




\begin{tabular}{cccccc}
\hline & & TT & $(99) 97.1$ & $(107) 100$ & \\
\hline \multirow{2}{*}{ rs12722491 } & allele & C & $(204) 100$ & $(224) 100$ & - \\
\cline { 2 - 6 } & genotype & CC & $(102) 100$ & $(107) 100$ & $-^{-}$ \\
\hline \multirow{2}{*}{ rs550805995 } & allele & C & $(204) 100$ & $(224) 100$ & - \\
\cline { 2 - 6 } & genotype & CC & $(102) 100$ & $(107) 100$ & - \\
\hline \multirow{2}{*}{ rs12722621 } & allele & G & $(204) 100$ & $(224) 100$ & - \\
\cline { 2 - 6 } & genotype & GG & $(102) 100$ & $(107) 100$ & - \\
\hline
\end{tabular}

Table 5. The frequency of $H M G B$ allele and genotype in Iranian multiple sclerosis patients and controls.

\begin{tabular}{|c|c|c|c|c|c|}
\hline Polymorphism & & Status & $\begin{array}{c}\text { (Frequency of } \\
\text { Patients) Percent }\end{array}$ & $\begin{array}{c}\text { (Frequency of } \\
\text { Control) Percent }\end{array}$ & P-value \\
\hline \multirow{4}{*}{ DELINSCrs146076135 } & allele & $\mathrm{C}$ & (183) 97.0 & (195) 70.0 & \\
\hline & & $\mathrm{C}-/-$ & (0) 0.0 & (1) 0.9 & \multirow{3}{*}{0.51} \\
\hline & genotype & $\mathrm{Cl}-$ & (21) 20.6 & $(27) 24.1$ & \\
\hline & & $\mathrm{C} / \mathrm{C}$ & (81) 79.4 & (84) 75.0 & \\
\hline \multirow{2}{*}{ DELINSTrs201945336 } & allele & $\mathrm{T}$ & (204) 100 & & - \\
\hline & genotype & $\mathrm{T} / \mathrm{T}$ & (102) 100 & (112) 100 & - \\
\hline \multirow{2}{*}{ rs111892138 } & allele & $\mathrm{A}$ & (204) 100 & & - \\
\hline & genotype & $\mathrm{A} / \mathrm{A}$ & (102) 100 & $(112) 100$ & - \\
\hline \multirow{2}{*}{ DELINSArs200308321 } & allele & A & (204) 100 & (214) 100 & \\
\hline & genotype & A-/A- & (102) 100 & $(107) 100$ & - \\
\hline \multirow{3}{*}{$\begin{array}{c}\text { DELINSGAAGrs14963710 } \\
8\end{array}$} & allele & GAAG & (204) 100 & (222) 91.0 & \\
\hline & & GAAG/- & (0)0.0 & (1) 0.9 & \multirow{2}{*}{0.98} \\
\hline & genoup & GAAG/GAAG & (102) 100 & (111)99.1 & \\
\hline \multirow{2}{*}{ rs538493533 } & allele & $\mathrm{T}$ & (204) 100 & $(224) 100$ & \\
\hline & genotype & TT & (102) 100 & (112)100 & \\
\hline \multirow{2}{*}{ rs577524260 } & allele & $\mathrm{G}$ & (204) 100 & $(224) 100$ & - \\
\hline & genotype & GG & (102) 100 & (112)100 & - \\
\hline \multirow{2}{*}{ rs182881863 } & allele & A & (204) 100 & $(224) 100$ & - \\
\hline & genotype & AA & 100 & (112)100 & - \\
\hline
\end{tabular}
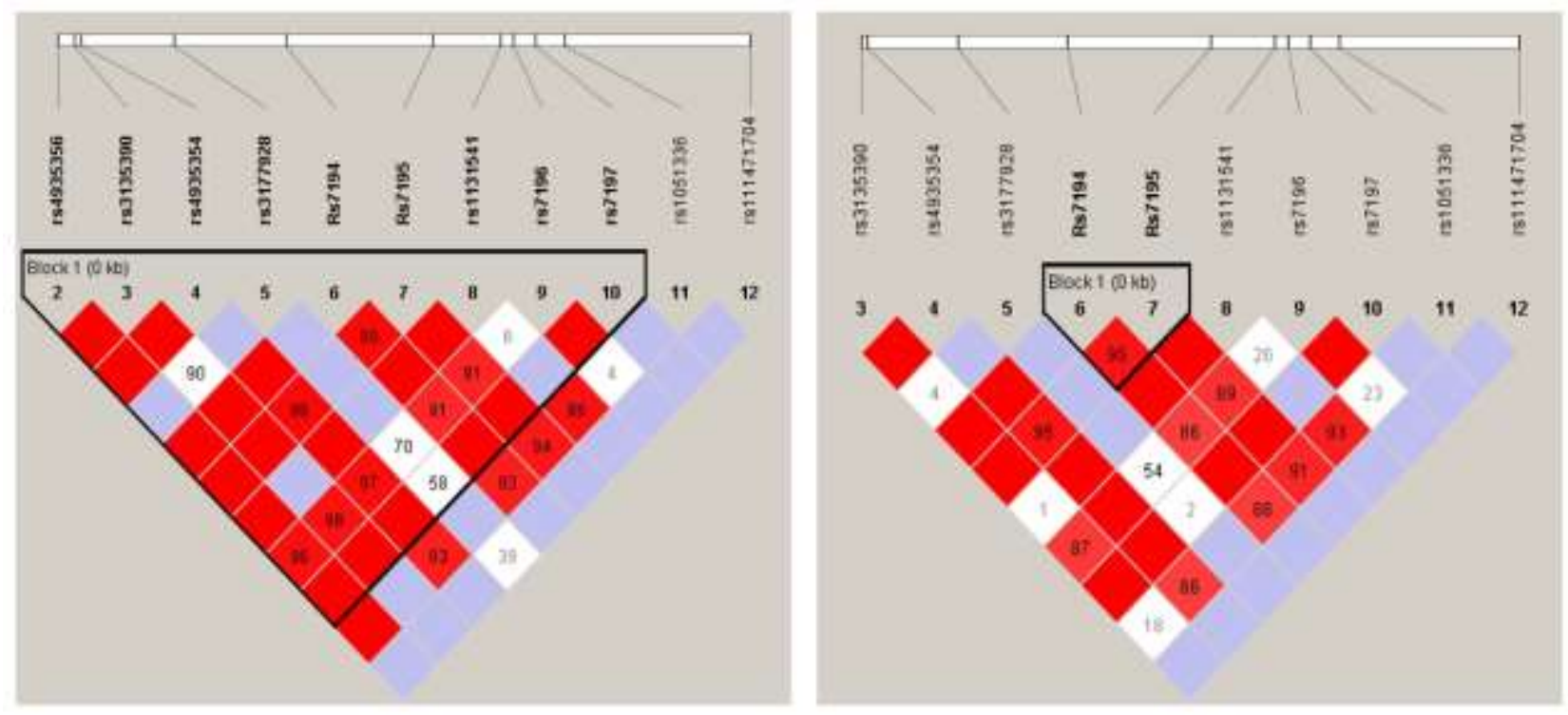

Fig. 1. Linkage disequilibrium (LD) of HLA-DRA SNPs using $D^{\prime}$ (left, red color) and $r^{2}$ (right, gray color) values for patient (left) and control (right) group. High levels of $\mathrm{LD}$ are depicted via increasing scale intensity from 0 to 100 , as exhibited by the bars. 


\section{Discussion}

In the present study, we investigated the associations between 36 SNPs and the risk of MS in a population in Iran. A significant association ( $\mathrm{p}<0.05)$ among certain SNPs from HLA-DRA and IL2RA genes were found. Although a number of non- $H L A$ genes have recently been recognized to contribute to susceptibility to MS with a modest effect, the $H L A$ region is generally identified as being the strongest risk contributor. Meta-analysis studies suggest that $H L A-D R 2$, and specifically the $D R B 1 * 15$ allele, are significant risk determinants in Chinese MS patients, nonetheless less strong correlations were revealed in Western MS populations; whereas, HLA-DR9 alleles appeared to confer the resistance to disease in this population (13).

This study showed significant associations as well as significant allele frequency differences in some of the studied SNPs including rs4935356, rs3177928, and rs7197 from the HLA-DRA gene and rs12722489 and rs12722490 variants from the IL2RA gene.

The rs4935356 variant from the HLA-DRA gene was previously reported to be involved in alcoholism and addiction disorders through influencing the brain, behavior, and immune system (5). Here, by observing significant associations, we found a novel relationship and possibly new disease-related function for rs4935356 at risk of MS condition. In addition, the SNP variant rs3177928, significantly related to increased risk of MS, is reported in novel $H L A$ $D R A$ downstream variants that were independent of $H L A-D R B 1$ alleles which are associated with non-Löfgren sarcoidosis (NL Sarcoidosis) as a multiorgan inflammatory disorder. Recent nonsarcoidosis studies showed that rs3177928 was associated with lipoprotein metabolism and connected with inflammatory mechanisms (14). Moreover, recently it was discovered that rs7197 SNP is strongly associated with antibody response against viral elements such as Epstein-Barr virus (EBV) capsid antigen. Our results are consistent with this finding and suggest that rs7197 genetic variation might have a role in developing MS disease through immune-mediated and particular microbial genes and susceptibility (15). In addition, the results of $\mathrm{LD}$ analysis demonstrated a strong linkage between rs7195 and rs7194, which was confirmed by findings showing almost the same level of non-significance for their genotypes and allele frequencies.

The IL-2/IL2R signaling stimulates the proliferation and survival of activated $\mathrm{T}$ cells and has a paramount non-redundant role in the production of regulatory $\mathrm{T}$ cells (16). Our data showed significant associations of rs12722489 and rs12722490 SNPs in IL2RA and the risk of MS. Additionally, allele frequency differences were noted too. The rs12722489 polymorphism is linked with multiple autoimmune conditions such as rheumatoid arthritis, multiple sclerosis, Crohn's disease, and ulcerative colitis. In silico analysis suggested significant discrepancies in the affinity of estrogen receptor (ER) binding site between the alternative allelic variants, with a stronger predicted affinity for the risk (G) allele. Electrophoretic mobility shift assessment illustrated that purified human ER $\alpha$ only bound $G$ variant of a 32-bp genomic sequence containing rs12722489 (17). Chromatin immunoprecipitation showed that endogenous ER $\alpha$ in humans interacted with rs12722489 genomic region in vivo and DNA pulldown assay confirmed differential allelic binding of amplified 189-bp genomic fragments containing rs12722489 with endogenous human ER $\alpha$. In a luciferase reporter assay, a kb-long genomic part containing $G$ but not A allele of rs12722489 demonstrated enhancer properties in MT-2 cell line, an HTLV-1 transformed human cell line with a regulatory $\mathrm{T}$ cell phenotype (17). Moreover, associations with various autoimmune disorders of polymorphisms in an LD block in which the IL2/IL21 genes map (4q27), and also in genes encoding the IL2RA and IL2RB subunits located in $10 \mathrm{p} 15$ and 22q13, respectively, were identified through GWAS. Polymorphisms in these three genes were studied in 430 MS patients and in 550 ethnically matched controls in Spain. Replication and meta-analysis with results from an independent cohort of 771 MS patients and 759 controls in Spain confirmed the association of polymorphisms in the IL2RA gene but did not verify the association for IL2RB (18). Regression analyses of the combined cohort in in Spain study revealed the independence of two IL2RA association signals: rs2104286 and rs11594656/rs35285258. The 
related role of the IL2RA gene on MS susceptibility is well in line with its common effect on autoimmune risk and the suggestive association of IL2/IL21 warrants further investigation (18).

The HMGBl belongs to the classification of endogenous damage-associated molecular pattern molecules (DAMPs), also known as alarmins. $H M G B 1$ is passively released from necrotic cells and it is actively secreted from activated immunocompetent cells, including macrophages (19). Nevertheless, extracellular HMGB1 has inhibitory effects on phagocytic activity of macrophages (efferocytosis), which is critical to the resolution of inflammation (20). Increasing evidence exists for the role of $H M G B 1$ in autoimmune disorders. In this context, recent studies have shown associations between $H M G B 1$ and rheumatoid arthritis, systemic lupus erythematosus, psoriasis, and Sjögren's syndrome (21). Studies on $H M G B 1$ are scarce in MS. In this examination, we considered the SNPs of $H M G B 1$; however, none of the studied polymorphisms was significantly associated with the risk of MS regarding both genotype and allele frequency. Zhen et al. examined $H M G B 1$ levels of peripheral blood mononuclear cells (PBMC), serum, and CSF in MS patients and found a considerably higher HMGB1 level in serum, PBMC, and CSF compared to healthy individuals and noninflammatory neurological disorder controls (10). Andersson et al. demonstrated that $H M G B 1$ and its receptors, RAGE (receptor for advanced glycation

\section{References}

1. Martin R, Sospedra M, Rosito M, Engelhardt B. Current multiple sclerosis treatments have improved our understanding of MS autoimmune pathogenesis. Eur J Immunol. 2016;46(9):2078-90. 2. Jamali M, Rostami Rad M, Anani Sarab G, Mahdavi R. IL-33 polymorphism rs1929992 and its association with susceptibility to different pattern of multiple sclerosis. Tehran Univers Med J. 2018;76(7):446-451.

3. Klaren RE, Sasaki JE, McAuley E, Motl RW, Health. Patterns and predictors of change in moderate-to-vigorous physical activity over time in multiple sclerosis. J Phys Act Health. 2017;14(3):183-188. end-products), are highly expressed in active lesions of MS as well as in its counterpart animal model EAE (experimental autoimmune encephalomyelitis), while being expressed at normal levels in inactive lesions. This suggests a potential interaction of these molecules in the inflammatory process involved in pathogenesis (11). We might suggest that the reason behind not significant differences between the two groups could be the effect of sample size or the fact that simply this genetic variation is not notably altered among Iranian MS patients.

In conclusion, we studied 36 SNPs in HLA$D R A, I L 2 R A$, and $H M G B 1$ genes in Iranian MS patients. Our results demonstrated significant associations of these genetic variants and MS disease. Further assessment of these SNPs in larger sample sizes along with other variants of mentioned genes would highly facilitate the recognition of causative variants in MS disease.

\section{Acknowledgment}

We are sincerely grateful to the staff of Shomal hospital at Amol, Iran and Mr. Morteza Gholami Ph.D. student at Metabolic Disorders Research Center, Tehran University of Medical Sciences and Dr. Mehrab Nasiri Kenari at North Research Center, Pasteur Institute of Iran for their technical assistance. We would like to thank the Education office, Pasteur Institute of Iran for financial support of this study.

The authors declare no conflict of interest.

4. Csepany T. Diagnosis of multiple sclerosis: A review of the 2017 revisions of the McDonald criteria. Ideggyogyaszati szemle. 2018;71(910):321-329.

5. Pan Y, Wang K-S, Wang L, Wu L-Y. Common Variants in HLA-DRA Gene are Associated with Alcohol Dependence in Two Caucasian Samples. J Mol Neurosci. 2013;49(3):574-81.

6. Olsson T, Barcellos LF, Alfredsson L. Interactions between genetic, lifestyle and environmental risk factors for multiple sclerosis. Nat Rev Neurol. 2017;13(1):25-36. 
7. Berge T, Leikfoss I, Brorson I, Bos S, Page C, Gustavsen $\mathrm{M}$, et al. The multiple sclerosis susceptibility genes TAGAP and IL2RA are regulated by vitamin $\mathrm{D}$ in CD4+ $\mathrm{T}$ cells. Genes Immun. 2016;17(2):118-27.

8. Fang P, Schachner M, Shen Y-Q. HMGB1 in development and diseases of the central nervous system. Mol Neurobiol. 2012;45(3):499-506.

9. Malhotra S, Fissolo N, Tintoré M, Wing AC, Castillo J, Vidal-Jordana A, et al. Role of high mobility group box protein 1 (HMGBl) in peripheral blood from patients with multiple sclerosis. J Neuroinflammation. 2015;12:48.

10. Zhen C, Wang Y, Li D, Zhang W, Zhang H, $\mathrm{Yu} \mathrm{X}$, et al. Relationship of High-mobility group box 1 levels and multiple sclerosis: A systematic review and meta-analysis. Mult Scler Rel Disord. 2019;31:87-92.

11. Andersson A, Covacu R, Sunnemark D, Danilov AI, Dal Bianco A, Khademi M, et al. Pivotal advance: $H M G B 1$ expression in active lesions of human and experimental multiple sclerosis. J Leukoc Biol. 2008;84(5):1248-55.

12. Buhelt S, Søndergaard HB, Oturai A, Ullum H, von Essen MR, Sellebjerg F. Relationship between Multiple Sclerosis-Associated IL2RA Risk Allele Variants and Circulating $\mathrm{T}$ Cell Phenotypes in Healthy Genotype-Selected Controls. Cells. 2019;8(6):634.

13. Wang S, Zhai H, Su Y, Wang Y. IL-17F but not IL-17A gene polymorphism confers risk to multiple sclerosis in a Chinese Han population. Journal of the Neurological Sciences. 2014;342(12):133-136.

14. Wolin A, Lahtela EL, Anttila V, Petrek M, Grunewald J, van Moorsel CH, et al. snP Variants in Major histocompatibility complex are associated with sarcoidosis susceptibility - a Joint analysis in
Four european Populations. Front Immunol. 2017;8:422.

15. Mentzer AJ, Brenner N, Allen N, Littlejohns TJ, Chong AY, Cortes A, et al. Identification of host-pathogen-disease relationships using a scalable Multiplex Serology platform in UK Biobank. medRxiv. 2019:19004960.

16. Mahmud SA, Manlove LS, Farrar MA. Interleukin-2 and STAT5 in regulatory $\mathrm{T}$ cell development and function. JAKSTAT. 2013;2(1):e23154.

17. Afanasyeva MA, Putlyaeva LV, Demin DE, Kulakovskiy IV, Vorontsov IE, Fridman MV, et al. The single nucleotide variant rs12722489 determines differential estrogen receptor binding and enhancer properties of an IL2RA intronic region. PLoS One. 2017;12(2):e0172681.

18. Cavanillas ML, Alcina A, Núñez C, De Las Heras V, Fernández-Arquero M, Bartolomé M, et al. Polymorphisms in the IL2, IL2RA and IL2RB genes in multiple sclerosis risk. European Journal of Human Genetics. 2010;18(7):794-799.

19. Erlandsson Harris $\mathrm{H}$, Andersson $\mathrm{U}$. Mini-review: the nuclear protein $H M G B 1$ as a proinflammatory mediator. Eur $\mathbf{J}$ Immunol. 2004;34(6):1503-12.

20.Friggeri A, Yang Y, Banerjee S, Park Y-J, Liu $\mathrm{G}$, Abraham E. HMGB1 inhibits macrophage activity in efferocytosis through binding to the avß3-integrin. Am J Physiol Cell Physiol. 2010;299(6):C1267-C1276.

21. Ek M, Popovic K, Erlandsson Harris H, Söderberg Nauclér C, Wahren-Herlenius $M$. Increased extracellular levels of the novel proinflammatory cytokine high mobility group box chromosomal protein 1 in minor salivary glands of patients with Sjögren's syndrome. Arthritis Rheum. 2006;54(7):2289-94. 